\title{
Comparison of silicon drift detectors made by Amptek and PNDetectors in application to the PHA system for W7-X
}

\author{
Natalia Krawczyk, \\ Jacek Kaczmarczyk, \\ Monika Kubkowska, \\ Leszek Ryć
}

\begin{abstract}
$\overline{\text { Abstract. The paper presents comparison of two silicon drift detectors (SDD), one made by Amptek, USA, and }}$ the second one by PNDetector, Germany, which are considered for a soft X-ray diagnostic system for W7-X. The sensitive area of the first one is $7 \mathrm{~mm}^{2} \times 450 \mu \mathrm{m}$ and the second one is $10 \mathrm{~mm}^{2} \times 450 \mu \mathrm{m}$. The first detector is cooled by a double-stage Peltier element, while the second detector is cooled by single-stage Peltier element. Each one is equipped with a field-effect transistor (FET). In the detector from Amptek, the FET is mounted separately, while in the detector from PNDetector, the FET is integrated on the chip. The nominal energy resolution given by the producers of the first and the second one is $136 \mathrm{eV} @ 5.9 \mathrm{keV}$ (at $-50^{\circ} \mathrm{C}$ ) and $132 \mathrm{eV} @ 5.9 \mathrm{keV}\left(\right.$ at $\left.-20^{\circ} \mathrm{C}\right)$, respectively. Owing to many advantages, the investigated detectors are good candidates for soft X-ray measurements in magnetic confinement devices. They are suitable for soft X-ray diagnostics, like the pulse height analysis (PHA) system for the stellarator Wendelstein 7-X, which has been developed and manufactured at the Institute of Plasma Physics and Laser Microfusion (IPPLM), Warsaw, in collaboration with the Max Planck Institute for Plasma Physics (IPP), Greifswald. The diagnostic is important for the measurements of plasma electron temperature, impurities content, and possible suprathermal tails in the spectra. In order to choose the best type of detector, analysis of technical parameters and laboratory tests were done. Detailed studies show that the most suitable detector for the PHA diagnostics is the PNDetector.
\end{abstract}

Key words: plasma diagnostic • pulse height analysis • solid-state detectors

N. Krawczyk ${ }^{\bowtie}$, J. Kaczmarczyk, M. Kubkowska, L. Ryć Institute of Plasma Physics and Laser Microfusion (IPPLM),

23 Hery Str., 01-497, Warsaw, Poland,

Tel.: +48 226381460 ext. 42, Fax: +48 226668372 ,

E-mail: natalia.krawczyk@ifpilm.pl

Received: 20 July 2015

Accepted: 5 January 2016

\section{Introduction}

The advanced stellarator Wendelstein 7-X, currently realized by IPP in Greifswald, is the next step in nuclear fusion research [1]. This device is going to demonstrate steady-state operation under plasma conditions relevant for a future fusion power plant. During the first operation phase OP1.1, the installed diagnostics will be checked and tested under stellarator conditions. One of them is the pulse height analysis (PHA) system which is foreseen to examine the soft X-rays emitted from the plasma during long pulse operation of W7-X [2]. This system was developed and manufactured at the Institute of Plasma Physics and Laser Microfusion (IPPLM), with the support and infrastructure provided by IPP, Greifswald [2]. The PHA diagnostic allows to investigate parameters such as the electron temperature, impurity content (e.g., C, Fe, Ni), as well as possible suprathermal tails in the spectra. However, in order to determine these parameters, the PHA system must be equipped with suitable detectors enabling efficient and effective operation. For this purpose, two silicon drift detectors (SDDs), one made by Amptek and a second one by PNDetector, 
have been considered and tested in the laboratory. Final results presented in this paper were decisive in choosing one of them.

\section{Application of silicon drift detectors for soft X-ray measurements}

The PHA system is a diagnostic that should provide the spectral energy distribution of emitted X-rays with an energy resolution high enough to separate all impurity spectral lines [3]. The system designed for W7-X is based on three energy channels (equipped with three SDDs), which allow to record an X-ray spectrum in three different energy ranges. With this solution, the sensitivity for impurity species increases. The first channel, features a very thin Polymer window, to be able to cover the energy range between $250 \mathrm{eV}$ and $20 \mathrm{keV}$. The second and third channel will be used to record spectra in the range 0.9-20 and $1.5-20 \mathrm{keV}$, respectively. In order to choose the proper energy range, depending on the experimental conditions, all channels are equipped with additional filters. To increase or decrease the photon flux, all channels have individual control of the pinhole (each pinhole consists of two piezo slits, which can be changed in the range $0-1.35 \mathrm{~mm}$ ). The efficiency of three energy channels are presented in Fig. 1.

The SDDs are suitable for low-energy X-ray spectroscopy because they combine a large sensitive area with a small value of the output capacitance [4]. Owing to this fact, they are the best candidate for the PHA diagnostics.

Thereby, these sensors are characterized by a higher resolution and count rate in comparison to other semiconductor detectors. Additionally, another benefit of these detectors is their cooling system based on the Peltier element. It allows to work at room temperature and thus avoids impractical liquid nitrogen cooling.

In the case of the PHA system, two different SDDs were considered. Both detectors are widely used in X-ray spectroscopy because of the numerous advantages that they guarantee. Selecting one of them was based on, i.a. matching technical parameters to the future PHA working conditions.

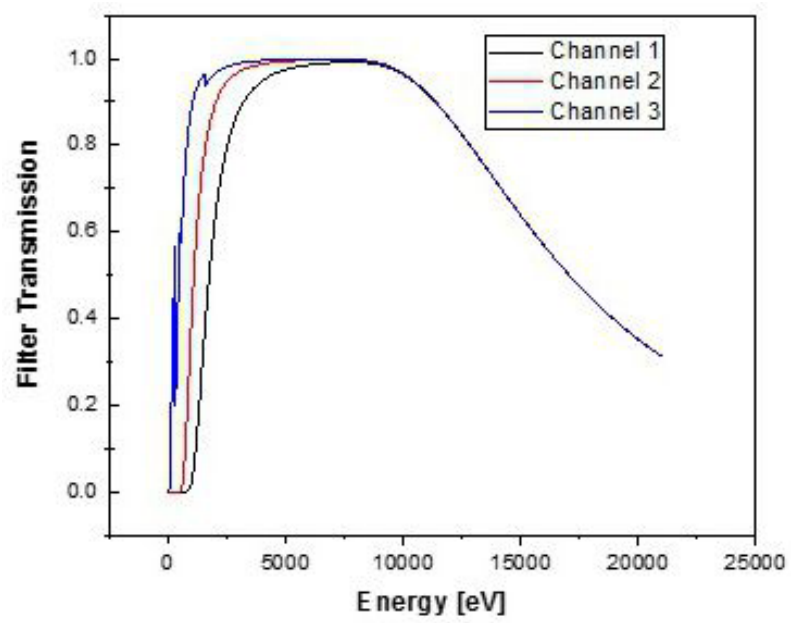

Fig. 1. Efficiency of three energy channels.
The first detector, made by Amptek (model XR-123SDD), has a silicon crystal with a thickness of $450 \mu \mathrm{m}$, an active area of $7 \mathrm{~mm}^{2}$, and a Be window (with thickness of $12.5 \mu \mathrm{m}$ ). This detector is cooled by double-stage Peltier element that sets the operating temperature to $-50^{\circ} \mathrm{C}$. The energy resolution given by the producer can achieve $136 \mathrm{eV}$ at $5.9 \mathrm{keV}$. The second detector from PNDetector (model SDD-10-130 BeW ic) with thickness of $450 \mu \mathrm{m}$ and an active area of $10 \mathrm{~mm}^{2}$ is equipped with an $8-\mu \mathrm{m}$ thick beryllium window. The crystal is cooled by single-stage Peltier element to around $-20^{\circ} \mathrm{C}$. In this case, the energy resolution given by the producer is $132 \mathrm{eV}$ at $5.9 \mathrm{keV}$. Both detector systems are equipped with a field-effect transistor (FET). However, their location in the sensor system is different. The SDD by Amptek uses the FET mounted separately, while the SDD from PNDetector has the FET integrated on the chip. In both cases, the application of the FET is very important because it contributes to improve the energy resolution.

\section{Laboratory test (experimental setup)}

Laboratory tests of the two detectors were carry out by using X-ray fluorescence (XRF) analysis. XRF is a widely used and non-destructive technique for the analysis of materials. This method is based on the generation of characteristic X-rays from a sample, which is irradiated by an X-ray tube or a radioactive source [5]. During this process, X-rays can be absorbed by an atom. Consequently, this leads to the ejection of an electron from the atom, thus creating vacancies. After this, in order to obtain a stable state again, an electron from the outer shell falls into the vacancy that is located in the inner shell. Then, as a result, the characteristic X-ray radiation is generated. Its energy is equal to the difference between the energy levels of outer and inner shells.

In the present laboratory tests, a mini X-ray tube (made by Amptek) was used as an excitation source. The features of this device are a silver $(\mathrm{Ag})$ transmission target, a beryllium end window, and a $40 \mathrm{kV} / 80 \mu \mathrm{A}$ power supply. The applied X-ray source was integrated with the PHA system.

A calibration was carried out by measurements of fluorescence spectra using well-known materials such as $\mathrm{Fe}, \mathrm{Cu}, \mathrm{Cr}$, and $\mathrm{Ni}$. These elements originate from a stainless steel plate with the addition of copper. The acquisition time was about $90 \mathrm{~min}$. The calibration procedure was performed separately for each of the two investigated detectors. This process allowed to verify the parameters of detector such as especially the full width at half maximum (FWHM).

In spectra registered with both detectors, seven lines derived from iron ( $\mathrm{Fe})$, nickel (Ni), chromium $(\mathrm{Cr})$, and copper $(\mathrm{Cu})$ are identified. These correspond to the two characteristic transitions $\mathrm{K} \alpha$ and $\mathrm{K} \beta$. The $\mathrm{K} \alpha$ lines (in presented case: $\mathrm{K} \alpha \mathrm{Cr}, \mathrm{K} \alpha \mathrm{Fe}$, $\mathrm{K} \alpha \mathrm{Ni}, \mathrm{K} \alpha \mathrm{Cu}$ ) correspond to transitions from the $\mathrm{M}$ and $\mathrm{N}$ shells to the $\mathrm{K}$ one, while the $\mathrm{K} \beta$ lines (in presented case: $\mathrm{K} \beta \mathrm{Cr}, \mathrm{K} \beta \mathrm{Fe}, \mathrm{K} \beta \mathrm{Cu}$ ) correspond to transitions from the $\mathrm{L}$ to the $\mathrm{K}$ shell. Examples of 

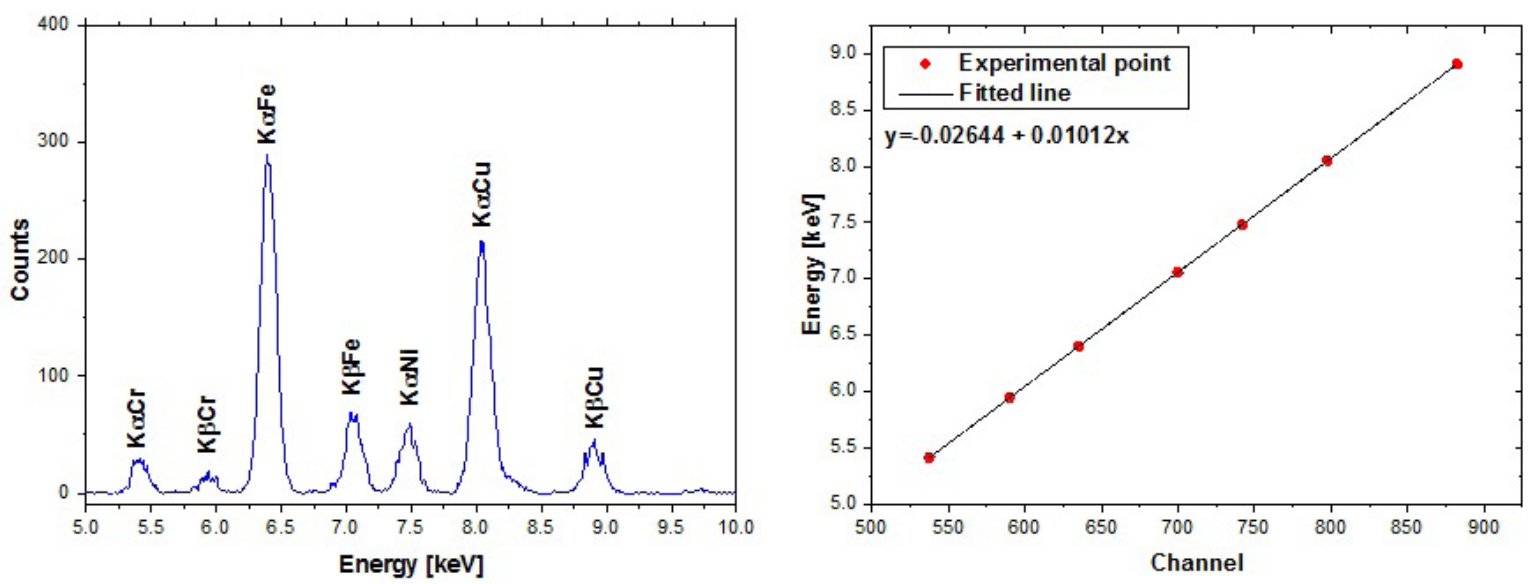

Fig. 2. Registered spectrum by the SDD from PNDetector (8- $\mu \mathrm{m}$ Be window) with calibration curve.
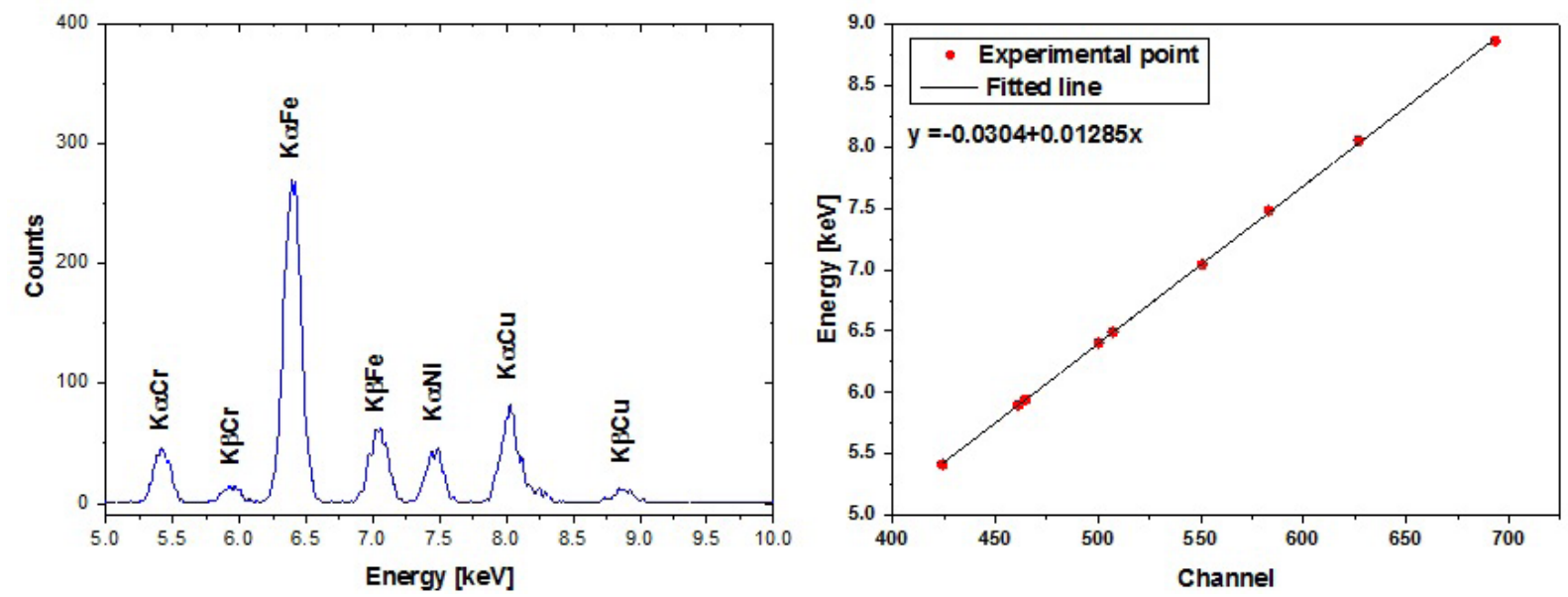

Fig. 3. Registred spectrum by the SDD from Amptek (12.5- $\mu \mathrm{m}$ Be window) with calibration curve.

these spectra are presented in Figs. 2 and 3. These results show that both the detectors work in a satisfactory manner because all expected lines were registered. The same figures show the calibration curves. The dependency between channel and energy is necessary to create another graph that gives the FWHM as a function of the energy range for each detector (Fig. 4). The obtained dependence shows that in the case of the sensor from PNDetector, the energy resolution increases with energy and its value is maintained at an average level of $123.600 \pm 0.003 \mathrm{eV}$. This dependence

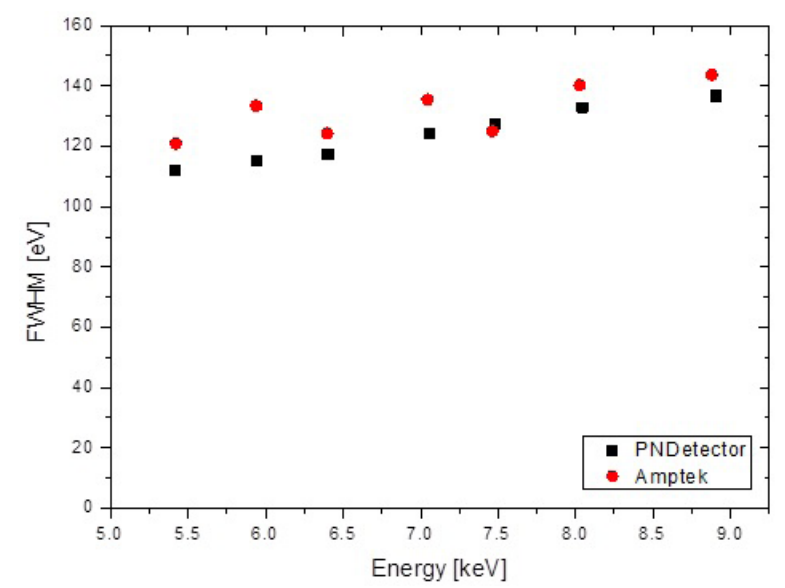

Fig. 4. The full width at half maximum (FWHM) as a function of energy range (for both detectors). did not occur in the detector from Amptek. Probably, this is connected with the measured spectrum in which the individual lines have jagged shape. Moreover, in the case of the sensor from the United States, the energy resolution is slightly worse - about $131.800 \pm 0.004 \mathrm{eV}$. Although both detectors were positive in this test, the SDD made by PNDetector was slightly better.

\section{Conclusions}

In the second half of 2015, the first experimental campaign on the Wendelstein $7-X$ took place. During the first operation phase OP1.1, the PHA system starts its work. The basis of the system will be three SDDs, which will allow to determine some important parameters from a plasma diagnostic point of view. Two detectors, one from Amptek and one from PNDetector, were tested in the laboratory, including an analysis of their technical parameters. The obtained results indicated that the SDD from PNDetector is sligthly better. The measurements of the fluorescence spectra show that its average energy resolution is about $123.600 \pm 0.003 \mathrm{eV}$ (the target contained $\mathrm{Fe}, \mathrm{Ni}, \mathrm{Cr}$, and $\mathrm{Cu}$ ). Moreover, its technical parameters such as single-stage Peltier element, FET integrated on the chip, as well as the optimum size of the chip $\left(10 \mathrm{~mm}^{2} \times 450 \mu \mathrm{m}\right)$ give an advantage 
over the similar detector from Amptek. Thereby, in the PHA system, three SDDs made by PNDetector (two with Be window and one with Polymer window) were used. This solution should provide stable and effective operation conditions of the system, already during the first operational phase of W7-X.

Acknowledgments. This work has been carried out within the framework of the EUROfusion Consortium and has received funding from the Euratom Research and Training Programme 2014-2018 under grant agreement no. 633053. The views and opinions expressed herein do not necessarily reflect those of the European Commission. This scientific work was partly supported by the Polish Ministry of Science and Higher Education within the framework of the scientific financial resources in the year 2014 and 2015 allocated for the realization of the international cofinanced project.

The work was performed at IPPLM in Warsaw, Poland.

\section{References}

1. Bosch, H. -S., Wolf, R. C., Andreeva, T., Baldzuhn, J., Birus, D., Bluhm, T., Bräuer, T., Braune, H., Bykov, V., Cardella, A., \& et al. (2013). Technical challenges in the construction of the steady-state stellarator Wendelstein 7-X. Nucl. Fusion, 53, 126001. DOI: 10.1088/0029-5515/53/12/126001.

2. König, R., Baldzuhn, J., Biel, W., Biedermann, C., Bosch, H. S., Bozhenkov, S., Bräuer, T., Brotas de
Carvalho, B., Burhenn, R., Buttehschön, B., Cseh, G., Czarnecka, A., Endler, M., Erckmann, V., Estrada, T., Geiger, J., Grulke, O., Hartmann, D., Hathiramani, D., Hirsch, M., Jabłonski, S., Jakubowski, M., Kaczmarczyk, J., Klinger, T., Klose, S., Kocsis, G., Kornejew, P., Krämer-Flecken, A., Kremeyer, T., Krychowiak, M., Kubkowska, M., Langenberg, A., Laqua, H. P., Laux, M., Liang, Y., Lorenz, A., Marchuk, A. O., Moncada, V., Neubauer, O., Neuner, U., Oosterbeek, J. W., Otte, M., Pablant, N., Pasch, E., Pedersen, T. S., Rahbarnia, K., Ryc, L., Schmitz, O., Schneider, W., Schuhmacher, H., Schweer, B., Stange, T., Thomsen, H., Travere, J. -M., Szepesi, T., Wenzel, U., Werner, A., Wiegel, B., Windisch, T., Wolf, R., Wurden, G. A., Zhang, D., Zimbal, A., Zoletnik, S., \& W7-X Team. (2015). The set of diagnostics for the first operation campaign of the Wendelstein 7-X stellarator. J. Instrum., 10, P10002.

3. Kubkowska, M., Czarnecka, A., Figacz, W., Jabłoński, S., Kaczmarczyk, J., Krawczyk, N., Ryć, L., Biedermann, C., Koenig, R., Thomsen, H., Weller, A., \& W7-X Team. (2015). Laboratory tests of the Pulse Height Analysis System for Wendelstein 7-X. J. Instrum., 10, P10016.

4. Lechner, P., Fiorini, C., Hartmann, R., Kemmer, J., Krause, N., Leutenegger, P., Longoni, A., Soltau, H., Stötter, D., Stötter, R., Strüder, L., \& Weber, U. (2001). Silicon drift detectors for high count rate X-ray spectroscopy at room temperature. Nucl. Instrum. Methods Phys. Res. Sect. A-Accel. Spectrom. Dect. Assoc. Equip., 458, 281-287.

5. El-Taher, A. (2012). Elemental analysis of granite by instrumental neutron activation analysis (INAA) and X-ray fluorescence analysis (XRF). Appl. Radiat. Isot., 70, 350-354. DOI: 10.1016/j.apradiso.2011.09.008. 